\title{
Swallowing Method of Administration
}

National Cancer Institute

\section{Source}

National Cancer Institute. Swallowing Method of Administration. NCI Thesaurus. Code C149973.

The technique of administration of a pharmaceutical product by introducing it into the stomach via the mouth and esophagus with a voluntary muscular action. 